\title{
The result of the study on double lumen tube anesthesia in Mongolian people
}

\author{
Bolormaa B ${ }^{1 *}$, Sanduijav R ${ }^{2}$, Avirmed $D^{3}$ and Lkhagvasuren TS \\ ${ }^{1}$ National Cancer Center, Mongolia \\ ${ }^{2}$ Medical Research Institute, Mongolia \\ ${ }^{3}$ Mongolian National University of Medical Sciences, Mongolia \\ ${ }^{4}$ Mongolian Academy of Medical Sciences, Mongolia
}

\begin{abstract}
In Mongolia, the morbidity and mortality rates of cancer have increased in the last decade and it became the second leading cause of death throughout the country. Therefore, the study has been conducted to evaluate this method of anesthesia in thoracic surgeries. Goal of the study: To determine the changes in arterial oxygenation and types of mechanical ventilation during the double lumen tube placement in anesthesia Mongolian thoracic surgery patients.
\end{abstract}

Abbreviations: One lung ventilation (OLV); Thoracic anesthesia (TA); Lung atelectasis (LA); Mechanical ventilation (MV)

\section{Materials and methodology of the study}

To meet the study inclusion criteria, total of 160 patients have been selected in this study and all of them underwent thoracic surgeries with the double lumen tubes in anesthesia, performed in NCC.

In this study the following materials and variables have been used, such as, general information of patients, complications occurred during and after the surgeries, duration of stay of patients in intensive care units, deaths, anesthesiology procedure, location of double lumen tubes and their size, volume of one or two lungs, arterial blood gas component of patients $\left(\mathrm{PaO}_{2}, \mathrm{PaCO}_{2}, \mathrm{SaO}_{2}, \mathrm{PH}\right)$, types of mechanical ventilation (CPAP, PEEP, PSV, PCV, ACV, CMV, SIMV).

\section{Result}

When consider diameter of the double lumen tubes, 35Fr were used in $78(48.75 \%)$ of 160 total patients and 37Fr were used in 82 patients (51.25\%). Positioning of double lumen tube in the tracheal bifurcation depends on the height of patients and we placed double lumen tube $27.68 \pm 2.47 \mathrm{~cm}$ deep in the tracheal bifurcation if patient $155 \mathrm{~cm}$ tall and $28.43 \pm 2.6 \mathrm{~cm}$ for $165 \mathrm{~cm}$ tall patient. According to the result of this study, we conclude that the left and right tracheal bifurcation length is estimated to be statistically significant $\mathrm{p}<0.004$ in Mongolian people. During the thoracic surgeries, degree of lung atelectasis was determined in 160 patients undergoing thoracic surgery and when open the chest, fully collapsed lung accounted for $84.3 \%$ (135 patients) and partially collapsed was $5.6 \%$ (9 patients) and the $10 \%$ of them was not inflatable.

During the one-lung ventilation, blood oxygen supply in the peripheral blood was $95.09 \%+1.07$ and $92.65 \%+5.69$ in arterial blood, $(\mathrm{p}<0.032)$. There were 91 patients $(56.8 \%)$ requiring increased respiratory rate during the one-lung ventilation with the double lumen tube, while 68 patients (43.2\%) did not need to change the respiratory rate during the two-lung ventilation.

During the one-lung ventilation with the double lumen tube, we have chosen an artificial type of respiration considering previous respiratory disease, age, and type of surgery. Pressure rate, volume, and air flow are the variables for choosing of mechanical ventilation. Both fully and supportive mechanical ventilation have been applied and $52.5 \%$ of them (84 cases) used during the surgeries and $17.75 \%$ (30 cases) used after the surgery.

\section{Conclusion}

As a result of the study, we have made a conclusion that it is necessary to place double lumen tube at $28.4 \mathrm{~cm}$ deep in the trachea if patient is $165 \mathrm{~cm}$ tall and position of the tube has to be adjusted by $0.78 \mathrm{~cm}$ in every $10 \mathrm{~cm}$ if patients is taller than $165 \mathrm{~cm}$. By using double lumen tube, operative site of thoracic surgery can be increased by 215 $240 \mathrm{~cm}^{3}$ and it prevents from the possible mechanical loads that would be occurred on the lung and heart.

During the thoracic surgery, where one lung isolated and other one is ventilated with double lumen tube, peripheral blood oxygen was examined. It was estimated to be $95.09 \%+1.07$ in peripheral blood and $92.65 \%+5.69$ in arterial blood, and the partial pressure of oxygen and carbon dioxide in the arterial blood was $<0.028$, proving that the patient had no oxygen deficiency during the operation.

During the double lumen tube anesthesia, fully mechanical (CMV, IPPV) and supportive ventilations (CPAP, SIMV, PEEP) have been used in of the study operation $(52.5 \%)$ and postoperative $(18.75 \%)$

${ }^{\star}$ Correspondence to: Bolormaa B, National Cancer Center, Mongolia, E-mail: batnasan_bolormaa@yahoo.com

Received: March 05, 2018; Accepted: March 16, 2018; Published: March 20, 2018 
period, alone болон or combination forms. As a result of the double lumen tube application, the operative and postoperative mechanical ventilation it is possible to prevent any difficulty in breathing or cardiovascular problems.

Copyright: $@ 2018$ Bolormaa B. This is an open-access article distributed under the terms of the Creative Commons Attribution License, which permits unrestricted use, distribution, and reproduction in any medium, provided the original author and source are credited. 